\title{
ОЦЕНКА ГОДНОСТИ ОБОРУДОВАНИЯ ЛОКОМОТИВОВ ПРИ ДОСТИЖЕНИИ ПОСЛЕ НОРМАТИВНОГО СРОКА ЭКСПЛУАТАЦИИ
}

\begin{abstract}
Александр Семёнович Крашенинин, д-р техн. наук., профессор кафедры «Эксплуатация и ремонт подвижного состава», механико-энергетического факультета

Елена Александровна Шапатина, ассистент кафедры «Управление грузовой и коммерческой работой»
\end{abstract}

Сергей Сергеевич Яковлев, аспирант кафедры «Эксплуатаџия и ремонт подвижного состава», механико-энергетического факультета

Украинский государственный университет железнодорожного транспорта; г. Харьков, Украина

DOI: https://doi.org/10.31435/rsglobal_ws/30042019/6466

\section{ARTICLE INFO}

Received: 10 February 2019

Accepted: 15 April 2019

Published: 30 April 2019

\section{KEYWORDS}

suitability, equipment,

locomotive,

criteria for assessing the shelf life,

changing the parameters of equipment operation.

\begin{abstract}
The article is devoted to the study of the assessment of the suitability of equipment locomotives in the the period after the end of the norms of operation based on the provisions of the theory of aging. It was determined that according to the minimization of the loss function of the axis of underutilization of equipment resources of locomotives, it is possible to extend their operation within 7-10 years, provided that this period is clarified for each specific equipment by the criterion of shelf life. This will give the opportunity to form the technology of Technical Service and Technical Regulations of equipment of locomotives after exceeding the standard lifetime.
\end{abstract}

Citation: А. С. Крашенинин, Е. А. Шапатина, С. С. Яковлев. (2019) Ocenka Godnosti Oborudovaniya Lokomotivov pri Dostizhenii Posle Normativnogo Sroka Ekspluatacii. World Science. 4(44), Vol.1. doi: 10.31435/rsglobal_ws/30042019/6466

Copyright: (C) 2019 А. С. Крашенинин, Е. А. Шапатина, С. С. Яковлев. This is an open-access article distributed under the terms of the Creative Commons Attribution License (CC BY). The use, distribution or reproduction in other forums is permitted, provided the original author(s) or licensor are credited and that the original publication in this journal is cited, in accordance with accepted academic practice. No use, distribution or reproduction is permitted which does not comply with these terms.

Цель исследования. Цель исследования заключается в определении предельных сроков эксплуатации локомотивов после достижения нормативного срока эксплуатации и уточнения срока продления эксплуатации оборудования локомотива. Уменьшение срока продления эксплуатации после достижения нормативного срока эксплуатации проведено на примере оценки коэффициента годности поршневой головки шарнира по параметру зазора на «масло». При решении задач оценки годности оборудования локомотивов использовались положения теории надежности для моделирования систем ремонта и критериальной оценки вариантов продления после нормативного периода эксплуатации локомотивов; методы теории старения для оценки годности и остаточного срока эксплуатации отдельного оборудования. Выполненные исследования по оценке годности оборудования локомотивов в период после нормативного срока эксплуатации от ранее проводимых исследований характеризуется комплексным подходом к уточнению остаточного срока использования оборудования локомотивов.

Введение. Важной составляющей расходов ресурсов на обеспечение жизненного цикла локомотивов являются затраты на их эксплуатацию. В сокращении этих затрат первичной 
составляющей является реализация оптимальной стратегии и тактики организации ремонта, поскольку от них зависят расходы на горюче-смазочные материалы, ущерб от нарушения регламента эксплуатационной работы и т.д. Ремонтная отрасль традиционно базируется на планово-предупредительных принципах организации, что в современных условиях не всегда в полной мере соответствует реалиям. Особенно это касается объемов и времени проведения необходимых ремонтных мероприятий в условиях старения оборудования локомотивов и устаревшей системы поставки запасных частей $[1,9,10,11]$.

В этих условиях одним из важных условий обеспечения эффективной работы ремонтного хозяйства является обоснование динамики изменения годности оборудования локомотивов для принятия решений по их дальнейшей эксплуатации или ее прекращения, что делает поставленную задачу актуальной.

Литературный обзор. Страны, эксплуатирующие тяговый подвижной состав нового поколения с высокотехнологичным оборудованием, в течение всего жизненного цикла стараются проводить комплекс мер по совершенствованию подходов по улучшению стратегии и тактики проведения технического обслуживания и текущего ремонта (ТО, ТР).

Так, японская железнодорожная компания [12] создала Центр анализа данных подвижного состава для организации мониторинга данных рабочего состояния оборудования, используемого в действующих поездах Токайдо Синкансен на основе анализа данных состояния подвижного состава. В исследовании [17] предложена методология оценки качества ремонта локомотивов на основе показателя энергоэффективности локомотива. В статье [19] отмечается, что значительный экономический эффект при организации ТО и ТР ремонта может быть достигнут путём реализации комплекса мер по совершенствованию планирования спроса и предложений, а также контроля материально-технических ресурсов для ремонта и технического обслуживания. В работе [15] обоснована методология корректировки технологических процессов для обеспечения гарантированного выполнения работ по подсистемам и агрегатам транспортных средств, которые оказывают наибольшее влияние на уровень аварийности. В работе [14] предлагается использование комбинированной системы поддержки железнодорожных двигателей, которые оснащены бортовыми диагностическими системами. В работе [13] сформулированы теоретические и практические направления улучшения качества ремонта на основе приведенных возможных вариантов организации технического обслуживания и ремонта железнодорожных двигателей. В статье [16]представлены результаты расчета показателей эффективности использования локомотивов для некоторых серий локомотивов. В работе [20] утверждается, что в зависимости от состояния подвижного состава планирование вариантов объёмов работ и технического обслуживания должно осуществляться по единому индексу надёжности. В работе [21] рассматривается разработка виртуальной системы обслуживания и ремонта подвижного состава, которая учитывает серию локомотива, состояние ремонтных участков депо и заводов, а также метод оценки организационно-технического уровня локомотиворемонтного депо.

Из приведенного краткого анализа можно отметить, что в Украине и за рубежом проведено достаточно много теоретических исследований, реализованных в практике работы локомотиворемонтных предприятий. В большинстве случаев эти исследования направлены на оптимизацию стратегии ТО, ТР локомотивов, что отражалось в оптимизации ремонтных циклов работы локомотивов и формировании тактики технологического обеспечения при ТО, TP $[1,10,11,12,13,14,16]$.

Особенность решения задач в реальных условиях эксплуатации заключается в дефиците ремонтного фонда и ремонтных мощностей. Исходя из этого, при формировании тактики ТО, ТР оборудования локомотивов следует учитывать динамику изменения годности этого оборудования.

Материалы и методы. Прогнозирование годности оборудования локомотивов осуществляется на основе обработки статистических данных с учётом различных распределений изменения их годности и оценки предельного срока после превышения нормативного срока нормативной эксплуатации локомотивов [1]. При решении задач оценки годности оборудования локомотивов использовались положения теории надёжности для моделирования системы ремонта и критериальной оценки вариантов продления после нормативного периода эксплуатации локомотивов; методы теории старения для оценки годности и срока эксплуатации отдельного оборудования. 
Определение особенностей эксплуатации тягового подвижного состава (ТПС) на всём этапе его жизненного цикла позволяет обосновать границы эффективности его использования.

Рассмотрим задачу определения оптимального срока эксплуатации ТПС с учетом экономической целесообразности его дальнейшего использования, в том числе и в посленормативный срок.

На оси времени обозначим точкой начало эксплуатации ТПС, и отдельными точками через установленные интервалы наработок (пробегов) - капитальный ремонт (рисунок 1). Обозначим начальную стоимость ТПС через $C_{0}$, а стоимость капитальных ремонтов через $C_{1}, C_{2}, C_{3}$. Промежуток времени работы до первого капитального ремонта обозначим через $t_{1}$, межремонтный срок между первым и вторым ремонтами $-t_{2}$, между вторым и третьим $-t_{3}$, и так далее.

Обозначим среднюю стоимость ТО и ТР, которые проводятся на один час работы в пределах первого периода через $\gamma_{1}$, в рамках второго периода через $\gamma_{2}$, и так далее. Сюда входит стоимость всех видов ТО и ПР до соответствующего капитального ремонта. Обозначим далее через $n_{1}, n_{2}, n_{3}$, - часовую производительность ТПС в соответствующих интервалах (рассчитанную, например, по стоимости единицы выполненной перевозочной работы).

Имея эти характеристики, можно получить выражение для средней себестоимости единицы производительности ТПС.

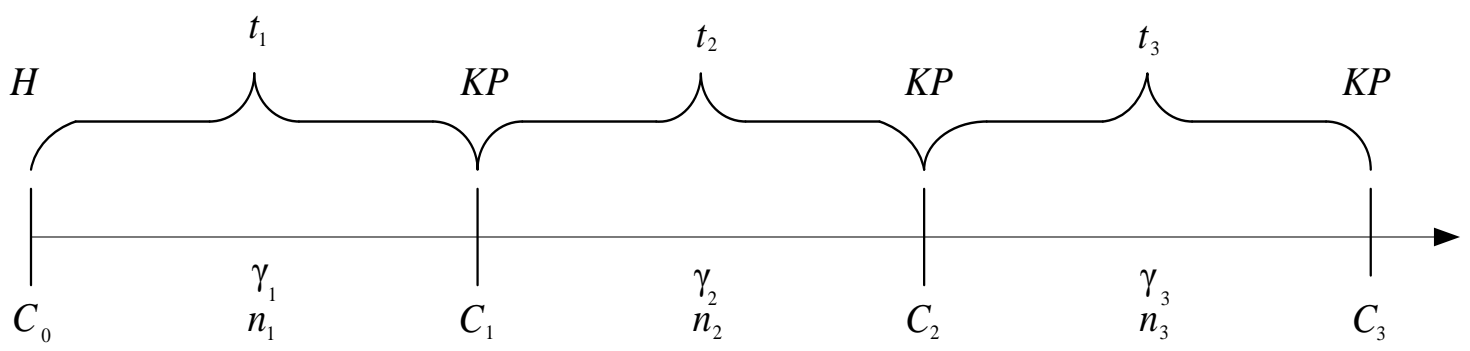

Рис. 1. Распределение расходов по основным этапам жизненного цикла

Для этого необходимо найти среднюю себестоимость $S_{1}$ в первом периоде от начала эксплуатации до первого капитального ремонта. Затем надо определить $S_{2}$ - среднюю себестоимость от начала эксплуатации до второго капитального ремонта; потом $S_{3}$ на период эксплуатации от начала до третьего капитального ремонта, и так далее.

Для этих средних себестоимостей можно записать следующие уравнения

$$
\begin{gathered}
S_{1}=\frac{C_{0}+\gamma_{1} t_{1}}{n_{1} t_{1}} \\
S_{2}=\frac{C_{0}+\gamma_{1} t_{1}+C_{1}+\gamma_{2} t_{2}}{n_{1} t_{1}+n_{2} t_{2}} \\
S_{3}=\frac{C_{0}+\gamma_{1} t_{1}+C_{1}+\gamma_{2} t_{2}+C_{2}+\gamma_{3} t_{3}}{n_{1} t_{1}+n_{2} t_{2}+n_{3} t_{3}+n_{1} t_{1}+n_{2} t_{2}+n_{3} t_{3}}
\end{gathered}
$$

или в общем виде для $n$-го этапа

$$
S_{n}=\frac{C_{0}+\sum_{i=1}^{n} \gamma_{i} t_{i}+\sum_{i=1}^{n-1} C_{i}}{\sum_{i=1}^{n} n_{i} t_{i}}
$$

где $C_{i}$ - стоимость $n$-го капитального ремонта.

Рассмотрим частный случай (который является весьма искусственным), когда стоимость всех капремонтов одинакова $C_{1}=C_{2}=C_{3}=\cdots$, грн.; также предположим, что межремонтные периоды, часовые производительности в межремонтный период и стоимости технического обслуживания также одинаковы: $t_{1}=t_{2}=t_{3}=\cdots$, часов; $n_{1}=n_{2}=n_{3}=$ $\cdots, 10^{4}$ ткмбр/час; $\gamma_{1}=\gamma_{2}=\gamma_{3}=\cdots$, грн./час; В этом случае из уравнений (1) - (3) получаем

$$
\begin{aligned}
& S_{1}=\frac{C_{0}}{n_{1} t_{1}}+\frac{\gamma_{1}}{n_{1}}, \text { грн./104 ткмбр(1a) } \\
& S_{2}=\frac{C_{0}+C_{1}}{2 n_{1} t_{1}}+\frac{\gamma_{1}}{n_{1}} \text {, грн./104 ткмбр } \\
& S_{3}=\frac{C_{0}+C_{1}+C_{2}}{3 n_{1} t_{1}}+\frac{\gamma_{1}}{n_{1}}, \text { грн./10 }{ }^{4} \text { ткмбр (3а) }
\end{aligned}
$$


Поскольку обычно $C_{1}<C_{0}$, то из этих уравнений получаем, что $S_{2}<S_{1}, S_{3}<S_{2}$ и так далее, то есть выгоднее все время выполнять капитальные ремонты, чем покупать новый ТПС. На самом деле так не бывает потому, что принятые выше предположения в эксплуатации невозможны. Так, первое предположение заключалось в том, что стоимость всех капитальных ремонтов одинакова. Обычно каждый последующий ремонт не проще предыдущего, и стоимость его будет больше. Постепенно с увеличением числа ремонтов, часовая производительность снижается. Межремонтные сроки тоже не остаются постоянными. Срок между началом эксплуатации и первым капитальным ремонтом обычно выше, чем между первым и вторым ремонтами. Межремонтные сроки эксплуатации ТПС корректируются на уменьшение. Стоимости ТО и ТР также не остаются постоянными. С увеличением наработки из-за старения, износа стоимость ТО и ТР постепенно растет, растут также затраты на горючесмазочные материалы, на единицу времени и тому подобное. Поэтому, реальная ситуация отличается от той, что описывается уравнениями (1a) - (3a).

Результаты. В качестве примера проиллюстрируем изменение средней себестоимости ТПС в течение жизненного цикла. Рассмотрим данные эксплуатации нового грузового электровоза постоянного тока ДЕ1, для которого по среднему суточным пробегом 350 км и средней массой состава поезда 5000 т примем3 возможных варианта изменения $n_{i}, \gamma_{i}, t_{i}$ при определении значения $S_{i}$.

В таблице 1 приведены расчёты средней себестоимости для трёх вариантов значений времени между ремонтами, часовой производительности ТПС и средней стоимости ТО и ТР. В первом случае все величины остаются постоянными, во втором случае изменяются часовая производительность и средняя стоимость ТО и ТР, а в третьем случае корректируется и промежуток времени работы между ремонтом, и часовая производительность, и средняя стоимость ТО и ТР. Промежутки времени между КР при корректировке межремонтных пробегов определялись исходя из опыта эксплуатации ТПС на Южной железной дороги в пределах: для второго интервала $t_{2}=0,9 * t_{1}$, для третьего интервала $t_{3}=0,85 * t_{1}$, для четвёртого интервала $t_{4}=0,8 * t_{1}$. Аналогично часовая производительность изменялась: $n_{2}=$ $0,95 * n_{1}, n_{3}=0,9 * n_{1}, n_{4}=0,85 * n_{1}$, а средняя стоимость ТО, ТР $\gamma_{2}=1,1 * \gamma_{1}, \gamma_{3}=1,21 *$ $\gamma_{1}, \gamma_{4}=1,33 * \gamma_{1}$ Промежутки времени между КР при корректировке межремонтных пробегов определялись исходя из эксплуатации ТПС.

Таблица 1. Расчёт средних себестоимостей ремонта ТПС для 3-х вариантов

\begin{tabular}{|c|c|c|c|c|c|c|c|c|c|c|c|c|}
\hline \multirow{3}{*}{ 苞 } & \multirow{2}{*}{\multicolumn{3}{|c|}{$\begin{array}{l}\begin{array}{c}\text { Время работы между } \\
\text { ремонтом, } t, \text { часов }\end{array} \\
\text { Первый вариант }\end{array}$}} & \multirow{2}{*}{\multicolumn{3}{|c|}{$\begin{array}{c}\text { Часовая } \\
\begin{array}{c}\text { производительность ТПС } \\
n, \mathbf{1 0}^{4} \text { ткм/час }\end{array} \\
\text { Второй вариант }\end{array}$}} & \multirow{2}{*}{\multicolumn{3}{|c|}{$\begin{array}{c}\text { Средняя стоимость ТО и } \\
\text { ТР, } \gamma, \text { грн./час } \\
\text { Третий вариант }\end{array}$}} & \multicolumn{3}{|c|}{$\begin{array}{c}\text { Средняя себестоимость, } \\
S_{i}, \text { грн./10 } \text { tк }^{4} \text { ткм }\end{array}$} \\
\hline & & & & & & & & & & \multirow[b]{2}{*}{ 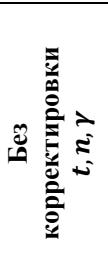 } & \multirow{2}{*}{ 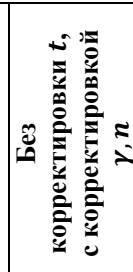 } & \multirow{2}{*}{ 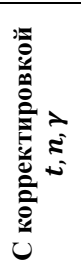 } \\
\hline & 总 & 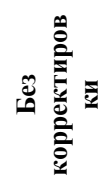 & 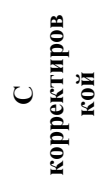 & 总 & U & 0 & 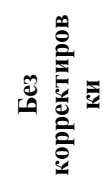 & U & 0 & & & \\
\hline КР-1 & 23000 & 23000 & 23000 & 15,7 & 15,7 & 15,7 & 120 & 120 & 120 & 21 & 21 & 21 \\
\hline KP-2 & 23000 & 23000 & 20700 & 15,7 & 14,92 & 14,92 & 120 & 132 & 132 & 15,9 & 16,8 & 17,2 \\
\hline КР-1 & 23000 & 23000 & 19500 & 15,7 & 14,13 & 14,13 & 120 & 145,2 & 145,2 & 15,0 & 17,29 & 17,3 \\
\hline КР-3 & 23000 & 23000 & 18400 & 15,7 & 13,35 & 13,35 & 120 & 159,7 & 159,7 & 14,2 & 17,5 & 17,4 \\
\hline
\end{tabular}

На рисунке 1 приведены графики изменения себестоимости для трёх вариантов 


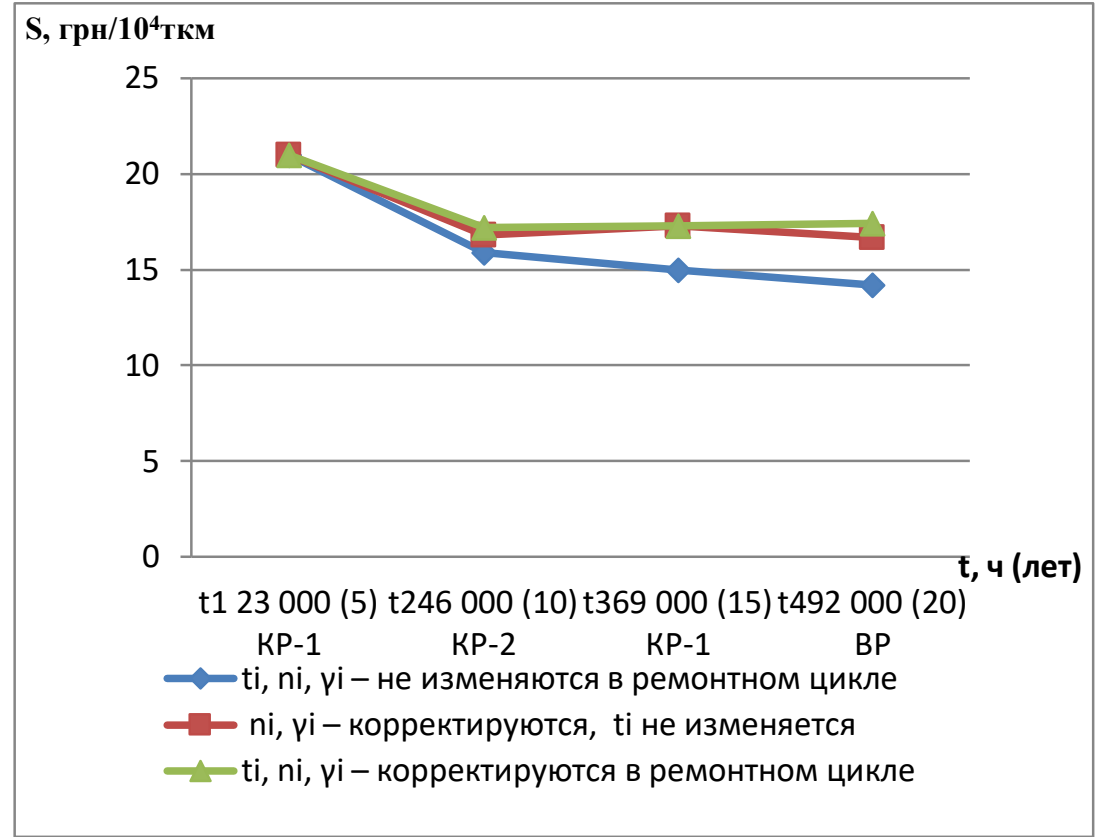

Рис.1 Изменение себестоимости $S_{i}$

Из расчетов, приведенных в таблице 1 и соответственно из графика на рисунке 1 видно, что сначала приведенная себестоимость является сравнительно высокой, поскольку при ее расчете учитывается стоимость нового ТПС. Затем себестоимость падает и затем снова начинает расти для второго и третьего вариантов. Графически это представлено на рисунке 1 , из которого видно, что в рассматриваемых случаях целесообразнее прервать эксплуатацию, когда появится необходимость проводить третий капитальный ремонт.

Вместе с тем в эксплуатации продолжается использование локомотивов, у которых превышено нормативное значение ресурса по отдельному оборудованию, что приводит к снижению надёжности и одновременно росту затрат на поддержание технического состояния оборудования.

Возникает необходимость определить остаточный и предельный посленормативные ресурсы по базовым узлам и агрегатам локомотивов, а после нормативный ресурс другого оборудования необходимо поддерживать за счёт корректировки ТО, ТР в период после нормативной эксплуатации. С учётом этого рассмотрим задачу таким образом, что необходимо определить ремонтный цикл такой продолжительности $L_{П Р}$, относительно которой итоговая разница между заданными $L_{1}, L_{2}, \ldots L_{N}$ значением ресурсов и искомыми $l_{1}, l_{2}, \ldots l_{N}$ межремонтными наработками оборудования достигнет минимума.

Потери от недоиспользования ресурсов оборудования определяются по формуле:

$$
H\left(L_{\Pi \mathrm{P}}\right)=\sum_{i=1}^{N}\left[\frac{C_{i}\left(L_{i}-l_{i}\right)}{L_{N}}-\frac{C_{i} \Pi_{i}\left(L_{i}-l_{i}\right)}{Ц_{\mathrm{T \Pi C}}}\right] \rightarrow \min ,
$$

где $\quad C_{i}$ - расходы на соответствующие ТО и ТР оборудования локомотива, грн./км;

$\Pi_{i}$ - средняя прибыль на выполненную работу, грн.;

Цтпс - стоимость локомотива, грн.

Упростим эту формулу, оставив её первую часть, при условии, что нас интересуют не приведенные на цену расходы, а оценка его обоснованного после нормативного срока службы.

Тогда перепишем формулу функции потерь таком виде

$$
H\left(L_{\mathrm{TP}}\right)=\sum_{i=1}^{N} \frac{L_{i}-l_{i}}{L_{N}} \rightarrow \min .
$$

Отметим, что при определении искомых значений ресурсов выполняется условие $l_{i} \leq L_{i}$.

Расчёты привяжем к долговечности базового оборудования локомотива, причём $L_{\Pi р}<$ $L_{N}$. Для простоты расчётов будем придерживаться кратности межремонтных пробегов $l_{i} / L_{i} N=$ $a_{i,}$ где $a_{i}=1,2, . .-$ целое число. 
Относительное значение $H\left(L_{\Pi \text { P }}\right)$, как критерий оптимальности ремонтного цикла, определяет долю итогового остаточного ресурса оборудования, которая приходится на единицу ресурса локомотива.

Минимальное значение этой величины характеризует предельный уровень ресурса, при котором его дальнейшее использование нецелесообразно и опасно.

Решение поставленной задачи заключается в определении значений $l_{1}, l_{2}, \ldots l_{N}$ наработки между заменами оборудования локомотива, которые минимизируют целевую функцию. При этом значение наработки базового оборудования локомотива определяет срок ремонтного цикла и соответствующее значение предельного ресурса при условии, что не предусматриваются дополнительные объёмы работ по оценке его технического состояния и состояния отдельного оборудования.

Оценить срок службы, за который локомотив реализует свой ресурс, можно по уравнению:

$$
T=\frac{L_{\Pi P}}{365 * S}
$$

где $S$ - значение среднесуточного пробега локомотива, км/сутки.

Для поиска оптимальных вариантов ремонтного цикла, который определяет обоснованный ресурс локомотива, будем придерживаться условий, которые приведены в приказе Укрзализныци №093, согласно положениям которого допускают пробеги до или после текущих ремонтов и КР в границах 20 \%. Для основных серий локомотивов 2ТЭ116, 2 ТЭ10М и 2TЕ10В значения этих пробегов приведены в таблице 1.

Таблица 2. Значения межремонтных пробегов для тепловозов 2ТЭ116, 2ТЭ10М, 2ТЭ10В

\begin{tabular}{|c|c|c|c|}
\hline \multirow{2}{*}{ Пробег, тыс. км } & Минимальный & Нормативный & Максимальный \\
\cline { 2 - 4 } & $L_{\min }$ & $L_{H}$ & $L_{\max }$ \\
\hline ТР-1 & 42,5 & 50 & 60,5 \\
\hline ТР-2 & 127,5 & 150 & 180 \\
\hline ТР-3 & 255 & 300 & 1080 \\
\hline КР-1 (1) & 720 & 900 & 2160 \\
\hline КР-2 & 1440 & 1800 & 1080 \\
\hline
\end{tabular}

В соответствии с этими значениями межремонтных пробегов были построены межремонтные циклы для 10 возможных вариантов их организации. На рис. 2 приведены два из вариантов межремонтного цикла. Другие варианты построены при условии изменения от $L_{\Pi р 1}=3000$ тыс.км до $L_{\Pi Р 10}=4500$ тыс.км. (максимальный суммарный срок службы после проведения второго КР-1). Для этих вариантов определены срок службы $T$ и предельный ресурс

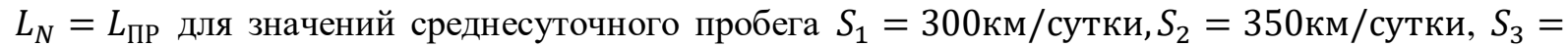
400км/сутки, или в относительных значениях: $\overline{L_{\mathrm{TP}}}=\overline{1 ; 1,5} ; \overline{S_{\text {Л }}}=1 ; 1,33$.

В таблицах 3, 4, 5 определено количество ТО, ТР для каждого из вариантов и соответствующие расходы на ТО, ТР, неплановые ремонты. В таблице 6 приведены значения $H\left(L_{\mathrm{TP}}\right)$ для этих вариантов ремонтного цикла.

Таблица 3. Срок службы локомотивов в зависимости от среднесуточного пробега

\begin{tabular}{|c|c|c|c|c|c|c|c|c|c|c|c|}
\hline \multicolumn{2}{|c|}{$L_{\Pi \text { Р, тыс. км }}$} & 3000 & 3100 & 3200 & 3300 & 3400 & 3700 & 3900 & 4100 & 4400 & 4500 \\
\hline \multirow{3}{*}{ 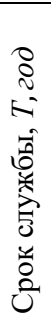 } & $\begin{array}{l}\mathrm{S}_{1} \\
=300 \text { км } \\
\text { /сутки }\end{array}$ & 27,4 & 28,31 & 29,22 & 30,14 & 31,05 & 33,79 & 35,62 & 37,44 & 40,18 & 41,10 \\
\hline & $\begin{array}{l}\mathrm{S}_{2} \\
=350 \text { км } \\
\text { /сутки }\end{array}$ & 23,48 & 24,27 & 25,05 & 25,83 & 26,61 & 28,96 & 30,53 & 32,09 & 34,44 & 35,23 \\
\hline & $\begin{array}{l}\mathrm{S}_{3} \\
=400 \text { км } \\
\text { /сутки }\end{array}$ & 20,55 & 21,23 & 21,92 & 22,60 & 23,29 & 25,34 & 26,71 & 28,08 & 30,14 & 30,82 \\
\hline
\end{tabular}



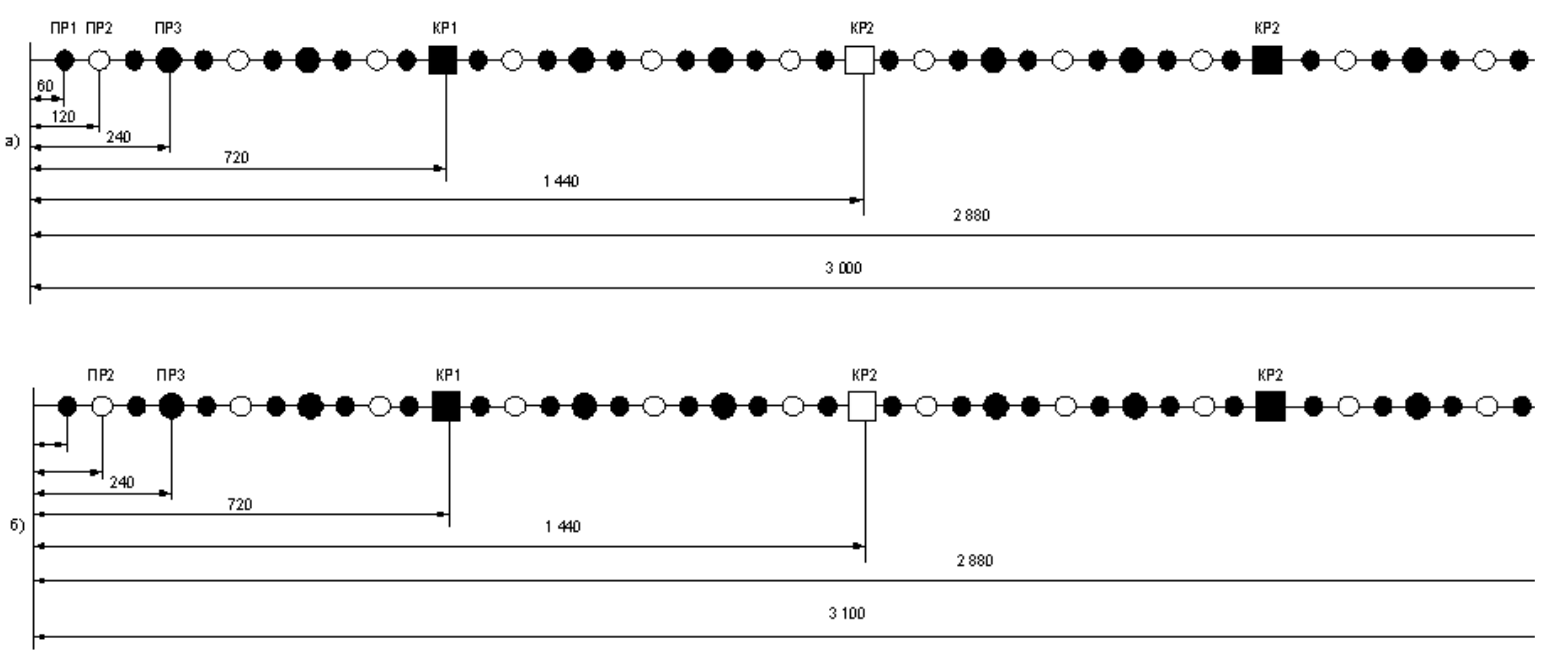

Pис. 2 Варианты межремонтных ичиклов (L, тыс. км)

Таблица 4. Расходы на плановый ремонт локомотивов

\begin{tabular}{|c|c|c|c|c|c|c|c|c|c|c|}
\hline $\begin{array}{c}L_{\text {ПР }} \text { тыс. } \\
\text { км }\end{array}$ & 3000 & 3100 & 3200 & 3300 & 3400 & 3700 & 3900 & 4100 & 4400 & 4500 \\
\hline$\sum_{\text {тыс.грн }} C_{\text {пл }}$ & 7815 & 7815 & 7695 & 7695 & 7695 & 8415 & 8775 & 8775 & 8295 & 8795 \\
\hline
\end{tabular}

Таблица 5. Расходы на неплановые ремонты

\begin{tabular}{|c|c|c|c|c|c|c|c|c|c|c|}
\hline $\begin{array}{c}L_{\text {ПР }} \text { тыс. } \\
\text { км }\end{array}$ & 3000 & 3100 & 3200 & 3300 & 3400 & 3700 & 3900 & 4100 & 4400 & 4500 \\
\hline$\sum_{\text {тыс.грн }} C_{\text {чл }}$ & 360 & 360 & 216 & 216 & 216 & 432 & 432 & 540 & 288 & 540 \\
\hline
\end{tabular}

Расчёты по неплановым расходам выполнялись по данным Южной железной дороги Украины из отчётных форм учёта затрат на неплановые ремонты депо

Таблица 6. Потери от недоиспользования ресурсов оборудования

\begin{tabular}{|c|c|c|c|c|c|c|c|c|c|c|}
\hline $\begin{array}{r}L_{\Pi Р}, \\
\text { тыс. км }\end{array}$ & 3000 & 3100 & 3200 & 3300 & 3400 & 3700 & 3900 & 4100 & 4400 & 4500 \\
\hline Расходь & 1. & 2. & 3. & 4. & 5. & 6. & 7. & 8. & 9. & 10. \\
\hline $\begin{array}{c}H\left(L_{\Pi Р}\right) \\
\text { при } S_{1}= \\
300 \text { км/ } \\
\text { сутки }\end{array}$ & 0,212 & 0,237 & 0,136 & 0,205 & 0,228 & 0,547 & 0,304 & 0,184 & 0,21 & 0,204 \\
\hline $\begin{array}{c}H\left(L_{\Pi Р}\right) \\
\text { при } S_{2}= \\
350 \text { км/ } \\
\text { сутки }\end{array}$ & 0,169 & 0,196 & 0,072 & 0,142 & 0,168 & 0,498 & 0,232 & 0,134 & 0,146 & 0,62 \\
\hline $\begin{array}{c}H\left(L_{\Pi Р}\right) \\
\text { при } S_{3}= \\
400 \text { км/ } \\
\text { сутки }\end{array}$ & 0,126 & 0,154 & 0,008 & 0,08 & 0,107 & 0,449 & 0,159 & 0,084 & 0,082 & 0,12 \\
\hline
\end{tabular}


Так, для первого варианта были приняты значения $l_{i}: 56 * 10^{3}$ км, $57 * 10^{3}$ км, $50 * 10^{3}$ км и соответственно определено значение $H\left(L_{\Pi Р}\right)$ по формуле

$$
\begin{aligned}
& H\left(L_{\Pi \mathrm{P}}\right)=\frac{\left(60-l_{i}\right) * 25+\left(120-2 * l_{i}\right) * 12+\left(240-4 * l_{i}\right) * 8}{3000} \\
& +\frac{\left(720-12 * l_{i}\right) * 2+\left(1440-24 * l_{i}\right) * 1+(3000-2880)}{3000}=0,212
\end{aligned}
$$

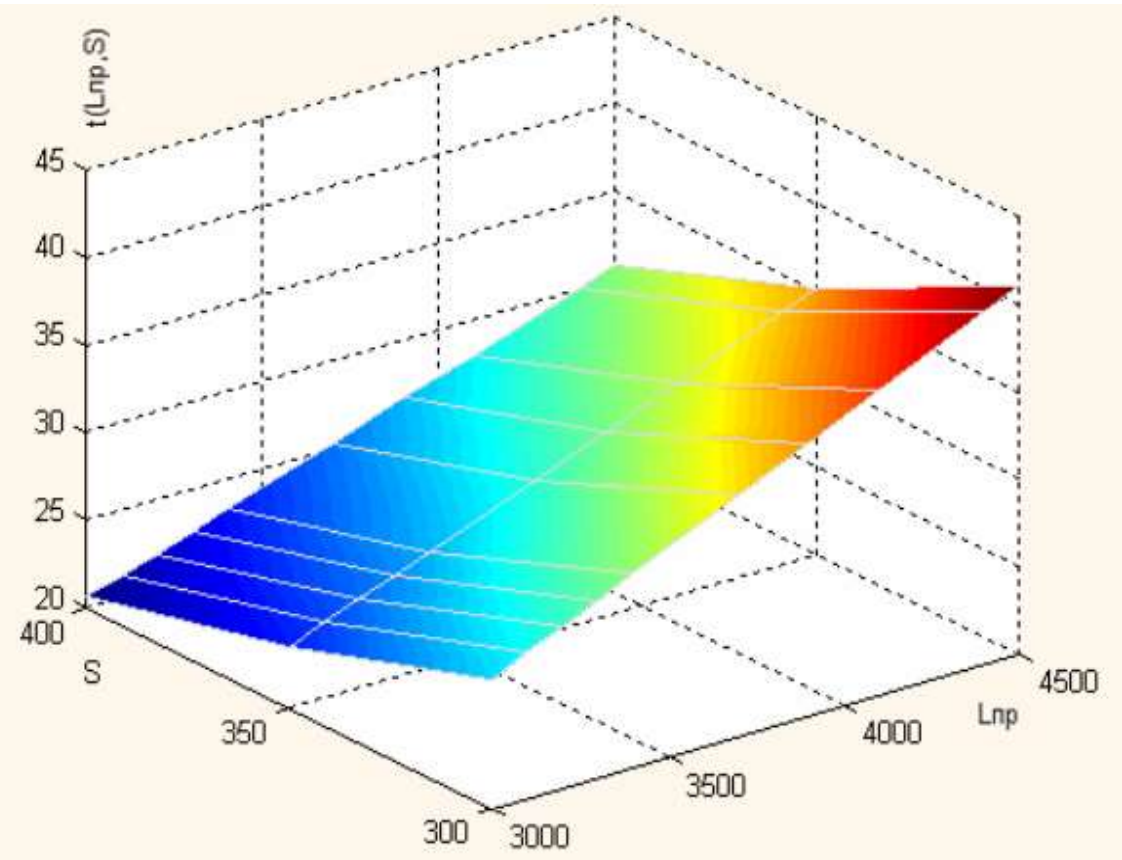

Рис. 3. Зависимость срока службы ТПС от пробега

На основании результатов этих расчётов на рис. 3,4 для рассмотренных вариантов построены зависимости $T\left(L_{\Pi \mathrm{P}}\right)$ и $H\left(L_{\Pi \mathrm{P}}\right)$. Минимум функции $H\left(L_{\Pi \mathrm{P}}\right)$ получен для варианта 3 $(T=22 \div 29$ лет), для 8 и 9 вариантов соответственно $T=28 \div 37$ лет и $T=30 \div 40$ лет.

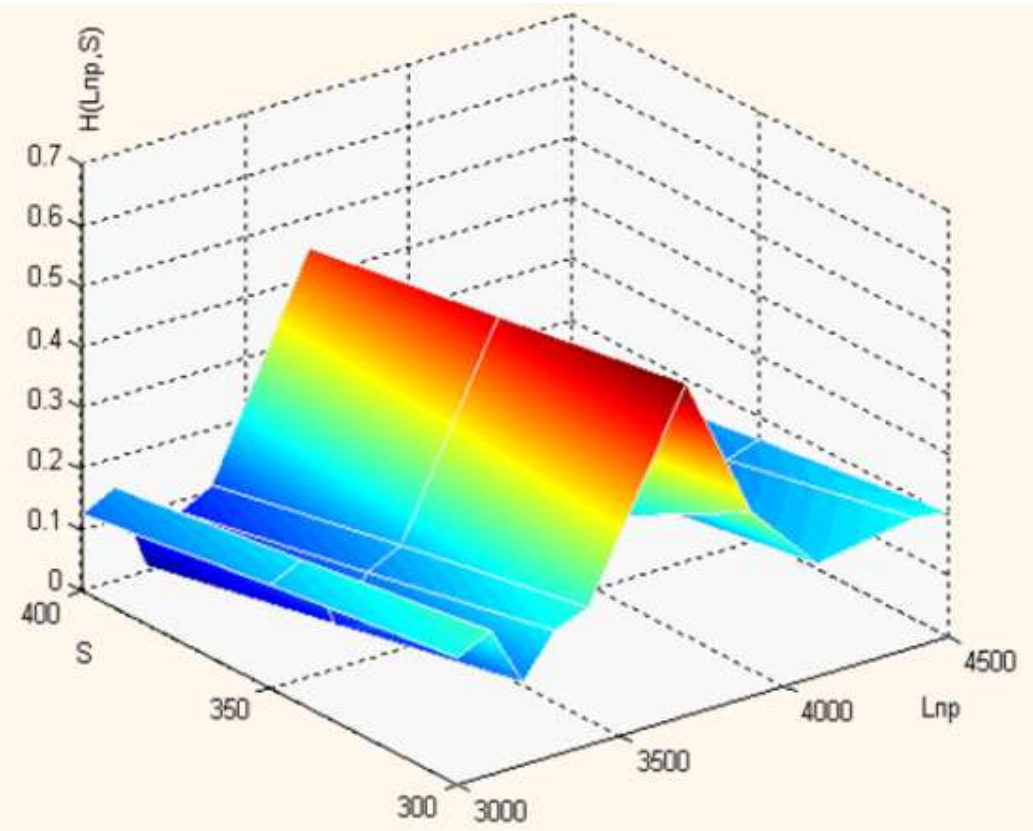

Рис. 4. Зависимость функции потерь от недоиспользования ресурса ТПС от пробега (срока службы) 
Продление срока эксплуатации по приведенному критерию определяет, что при соответствующих условиях его можно продлить с 22 до 40 лет, поскольку ресурс локомотивов зависит от интенсивности использования (величины среднесуточного пробега $S_{\mathrm{c}}$ ) и может изменяться в границах $7 \div 10$ лет.

Приведенные выше исследования показывают общин тенденции, характеризующие целесообразность продления срока эксплуатации ТПС после достижения нормативного срока эксплуатации.

Вместе с тем для принятия решения о завершении или продлении срока эксплуатации оборудования локомотива необходимо представлять каким остаточным ресурсом обладает конкретный технический объект. Нормативная литература определяет остаточный ресурс как наработку, после которой эксплуатация технического объекта должна быть прекращена.

Наглядным и эффективным инструментом оценки остаточного ресурса и уровня состояния технического объекта является оценка его годности, позволяющая обосновано принимать решения о завершении этапа эксплуатации. То есть при превышении нормативного срока эксплуатации локомотива следует оценивать среднее значение годности конкретного узла и его остаточный ресурс.

С целью упрощения процесса анализа изменения годности агрегатов и узлов локомотивов, сопоставления характеристик динамики параметров их технического состояния необходимо привести различные аппроксимирующие функции к канонической, базовой с помощью использования для получения уравнения годности обобщенного количественного показателя годности агрегатов и узлов локомотивов. Для этого проводится соответствующая обработка исходных данных $[2,4,5,6]$.

Наглядным и удобным методом подбора вида функций является нанесение эмпирических параметров на вероятностную бумагу. При совпадении или несовпадении нанесенных параметров с теоретической функцией, которая на вероятностной бумаге представляет собой линейную зависимость, принимаемое решение о правильности подобранной функции изменения годности технического объекта.

Процесс выбора функции годности состоит из двух этапов.

На первом этапе данные измерений наносят на сетку прямоугольных координат, соединяют экспериментальные точки плавной кривой и выбирают ориентировочно вид формулы.

На втором этапе вычисляют параметры функций, которые соответствовали бы принятому виду функции.

Процедуру реализации приведенных выше положений проиллюстрируем на примере оценки годности поршневой головки шатуна дизеля Д100 по параметру зазор «на масло». Отметим, что данная процедура аналогична для любой детали, узла или агрегата локомотива.

Как показали данные контроля изменения зазор на «масло» в поршневой головке шатуна дизеля $10 Д 100$ характеризуется следующими данными: вид закона распределения нормальный с параметрами $a_{0}=0,205$ мм, $\delta_{0}=0,033$ мм [2].Здесь $a_{0}$ - нормативное значение зазора «на масло». согласно правилам ремонта, $\delta_{0}$ - среднее квадратическое отклонение зазора на «масло» [2]. Случайный процесс изменения параметра можно описать уравнением

$$
U(l)=a_{0}+a e^{V_{l} l} \text {. }
$$

Уравнение показывает, что с течением времени нормативный параметр $a_{0}$ зазор «на масло» увеличивается из-за износа сопряженных поверхностей нелинейно.

Предельный зазор на «масло» в соответствии ремонтной документацией равен $U_{\Pi}=0,037$ мм. Результатами контроля зазора на «масло» $U_{i}(l)$ и соответствующая наработка $l_{i}$, тыс.км приведены в таблице 2. Наработки $l_{i}$ выбирались поотчетным данным деповской ремонтной документации локомотивного депо Основа Южной железной дороги за период 2014 - 2016 года. 
Таблица 6.

\begin{tabular}{|c|c|c|c|c|c|c|c|}
\hline $\begin{array}{c}\text { № } \\
\Pi / \Pi\end{array}$ & $\begin{array}{c}\text { Зазор } \\
U_{i}(l), \\
\text { мм }\end{array}$ & $\begin{array}{c}\text { Зазор } \\
U_{i}(l)-a_{0}\end{array}$ & $\begin{array}{l}\text { Наработка } \\
l_{i}, \text { тыс.км }\end{array}$ & $\frac{U_{i}(l)}{a}$ & $\ln \left(\frac{U_{i}(l)}{a}\right)$ & $\Gamma_{U_{i}}=\frac{\ln \left(\frac{U_{i}(l)}{a}\right)}{\ln \left(\frac{U_{\Pi}}{a}\right)}$ & $L_{i}=\frac{l_{i}}{\Gamma_{U_{i}}}$ \\
\hline 1 & 2 & 3 & 4 & 5 & 6 & 7 & 8 \\
\hline 1 & 0,208 & 0,003 & 130 & 2750,8 & 7,92 & 0,932 & 139,5 \\
\hline 2 & 0,207 & 0,001 & 150 & 2737,7 & 7,915 & 0,932 & 161,0 \\
\hline 3 & 0,208 & 0,003 & 160 & 2750,8 & 7,92 & 0,932 & 171,6 \\
\hline 4 & 0,215 & 0,010 & 180 & 2843,4 & 7,96 & 0,936 & 192,3 \\
\hline 5 & 0,240 & 0,035 & 210 & 3174,0 & 8,06 & 0,949 & 221,3 \\
\hline 6 & 0,260 & 0,055 & 220 & 3438,5 & 8,148 & 0,958 & 229,6 \\
\hline 7 & 0,270 & 0,065 & 230 & 3570,8 & 8,181 & 0,963 & 238,9 \\
\hline 8 & 0,290 & 0,085 & 250 & 3835,3 & 8,252 & 0,971 & 257,4 \\
\hline 9 & 0,320 & 0,115 & 270 & 4232,0 & 8,35 & 0,983 & 274,7 \\
\hline 10 & 0,360 & 0,155 & 300 & 4761,0 & 8,468 & 0,997 & 300,9 \\
\hline
\end{tabular}

Как видно из приведенных в таблице 6 данных, характер изменения зазора на «масло» в поршневой головке шатуна дизеля подтверждает представление случайного процесса в виде $U(l)=a_{0}+a e^{V_{l} l}$ (рис.5), что подтверждается видом этого процесса на вероятностной бумаге (рис.6) $[2,5]$.

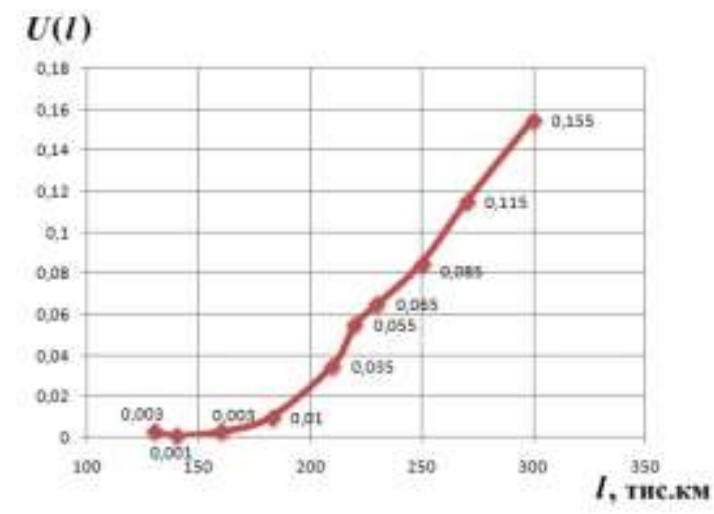

Рис.5 Изменение зазора на «масло» в поршневой головке шатуна дизеля $10 Д 100$ от пробега

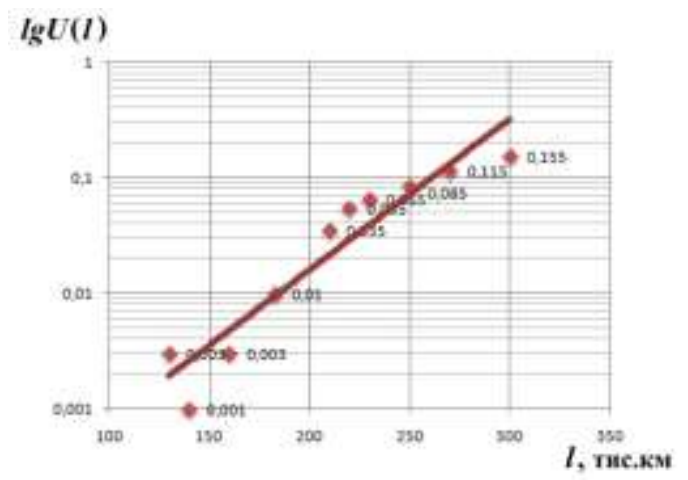

Рис.6 Изменение зазора на «масло» в поршневой головке шатуна дизеля $10 Д 100$ от пробега на вероятностной бумаге

Поскольку параметр $a_{0}=$ const, то логарифмирование уравнения (6) производилось по $\left[U(t)-a_{0}\right]=a e^{V i_{t}}$, т.e.

$$
\ln (U(t)-a)=\ln a+\mathrm{V}_{\mathrm{t}} \mathrm{t}
$$

Из этого уравнения по графику (рис. 6) можно определить по оси ординат значения свободного члена $\ln a$ и по антилогарифму значении $a$ и коэффициент увеличения зазора «на масло» $V_{t}$, как $\operatorname{tg} \alpha$ угла наклона построенной линейной зависимости.

Значения параметров $a$ и $V_{l}$ также определялись путем решения системы уравнений.

$$
\left\{\begin{array}{l}
\ln 0,015=\ln a+270 * V_{l} \ln l \\
\ln 0,01=\ln a+180 * V_{l} \ln l
\end{array}\right.
$$

Полученные значения коэффициентов соответственно равны $a=7,56 \cdot 10^{-5}$ мм; $V_{l}=0,027$ мм/тыс.км. Тогда изменение зазора на «масло» можно представить в виде 


$$
U(l)=0,205+7,56 \cdot 10^{-5} \cdot e^{V_{l} l} .
$$

В таблице 6 в столбцах 2 и 3 приведены значения изменения параметра зазора на «масло» в общем и приведенном виде для соответствующей наработки. Приведенная функция помещена в колонке 5, а логарифмированная - в колонке 6 как величина $\ln \left(\frac{U_{\Pi}}{a}\right)$.

В результате определяем ряд значений $\Gamma_{U_{i}}$ и наработку по каждой поршневой головке шатуна по параметру зазор на «масло».

Среднее значение годности поршневой головки шатуна по параметру зазора на «масло» равно:

$$
\bar{\Gamma}_{U}=\frac{\sum_{i=1}^{n} \Gamma_{U_{i}}}{n}=\frac{9,55}{10}=0,955,
$$

а средняя наработка

$$
I=\frac{\sum_{i=1}^{n} l_{i}}{n}=\frac{2187,1}{10}=218,7 \text { тыс.км. }
$$

Повышение точности расчётов можно достичь наряду с применением базовой аппроксимирующей функции более детальным учетом свойств условий работы узлов и агрегатов, то есть учетом характеристик функций скорости изменения параметров.

Таким образом, оценив для конкретных условий эксплуатации локомотивов после нормативный период его работы, необходимо поддерживать его путём контроля по изменению годности отдельных узлов и агрегатов, и средней наработки до исчерпания ресурса. В свою очередь, это будет предполагать проведение специальных дополнительных работ, если отдельные узлы имеют наработку меньше, чем у локомотива в целом.

\section{Обсуждение.}

1. Приведенные положения в отличии от цитируемых в разделе «Литературный обзор» имеют целью показать, что процесс оценки продления срока эксплуатации оборудования локомотивов предполагает определение предельного срока эксплуатации с учётом интенсивности работы локомотивов и уточнение его по коэффициенту годности анализируемого оборудования. Значение этого коэффициента в сочетании с оценкой средней наработки до списания позволит формировать тактику технических мероприятий для содержания локомотива. В условиях старения локомотивного парка и ремонтной инфраструктуры на фоне стагнации локомотивостроения это даст возможность обоснованию формировать стратегию определения годности оборудования после продления нормативного срока эксплуатации.

2. Необходимым этапом оценки годности является определение функции развития параметра, поиск значений коэффициентов которой следует выполнять с применением аналитических или графических методов.

3. Для каждой системы, агрегата, сопряжённых деталей представление развития деградации параметров безусловно требует определения своей математической модели и коэффициентов, её характеризующих. Это позволит сформировать базу данных для принятия решений о техническом содержании локомотивного оборудования в после нормативный период эксплуатации.

4. В отличии от рассматриваемых подходов в разделе «Литературный обзор» предлагаемый метод позволяет определить по статистике динамику годности оборудования локомотивов и среднюю наработку до списания.

Заключение. Проведенные исследования показывают, что в условиях старения парка ТПС существующая стратегия его содержания не в полной мере соответствует необходимым требованиям надёжного и эффективного его использования, особенно при продлении срока эксплуатации после достижения нормативного срока. Изучаемые подходы определяют целесообразность оценки посленормативного срока эксплуатации ТПС исходя из интенсивности его использования (среднесуточного пробега), необходимость корректировки технологии ТО, ТР для узлов и агрегатов по изменению их годности. Это потребовало необходимость обеспечения мониторинга технического состояния всех его узлов и агрегатов. В условиях дефицита запасных 
частей и агрегатов получение информации о динамике годности оборудования позволяет решать оперативные ремонтные задачи его содержания. Областью применения полученных результатов может быть и системы содержания других видов транспорта. Возможность также использовать методологические подходы в развитии заключительного этапа жизненного цикла организации системы утилизации отработанного оборудования ТПС.

\section{ЛИТЕРАТУРА}

1. Тартаковский Э.Д. Методы оценки жизненного цикла тягового подвижного состава железных дорог: Монография / Э.Д Тартаковский, С.Г. Грищенко, Ю.Е. Калабухин, А.П. Фалендыш. Луганск: Изд-во «Ноулидж», 2011. - 174 с.

2. Степнов М.Н. Статистические методы обработки результатов механических испытаний: Справочник. - М., «Машиностроение», 1985.

3. Михлин В.М. Прогнозирование технического состояния машин. М., «Колос», 1976

4. Большев Л.Н., Смирнов Н.В. Таблицы математической статистики. М., «Наука», 1983. - 416 с.

5. Джонсон Н., Лион Ф. Статистика и планирование эксперимента в технике и науке. Методы планирования эксперимента; Перевод с англ. / Под ред. Э.К. Лецкого, Е.В. Марковой. М., «Мир», 1981, $516 \mathrm{c}$.

6. Крамер Г. Математические методы статистики; Пер. с англ. / Под ред. А.Н. Колмогорова. М., «Мир», 1975, 648 с.

7. Смирнов Н.В., Дунин-Барковский И.В. Курс теории вероятностей и математической статистики для технических приложений. . М., «Наука», 1965, 511 с.

8. Степнов М.Н. Статистическая обработка результатов механических испытаний. . М., «Машиностроение», 1972, 232 с.

9. Крашенинин О. С., Шапатина О. О., Черняк Ю. В. и другие. Улучшение организации технического обслуживания и текущего ремонта тягового подвижного состава в после нормативный срок службы. транспортные инновации № 9, Киев, 2011.-с.26-28

10. Крашенинин А. С., Шапатина О. А., Матвиенко С. А., Зезюлин К. А. Оценка периодичности технического обслуживания и ремонта в период после нормативных сроков эксплуатации ТПС. Зб. наук. праць ДонІ3Т, 2011.-Вип. 128.- с.165-167

11. Крашенинин О. С., Харламов П. О. Оценка эффективности системы продления срока службы ТПС более нормативного и обновление эксплуатационного парка. Вестник Восточноукраинского университета им. Владимира Даля. Научныйжурнал № 3(174), Луганск, 2012. - c.109-113

12. Nishimura, K., Itoyama, M., Utano, A., Shinkansen Operations Division, Rolling Stock Department, Central Japan Railway Company, Japan. Establishment of inspection and repair system based on equipment data in Shinkansen rolling stock(Short Survey). Japanese Railway EngineeringVolume 2017January, Issue 195, January 2017, Pages 12-14

13. Bodnar B., Ochkasov, O., Dnipropetrovsk National University of Railway Transport Academician v. Lazaryana, Ac. Lazaryana Str, 2, Dnipro, Ukraine. System choice of the technical maintenance of locomotives equipped with on-board diagnostic systems(Conference Paper). Transport Means Proceedings of the International Conference2017, Pages 43-4721st International Scientific on Conference Transport Means 2017; Rest House "Azuolynas"L. Rezos Str. 54Juodkrante; Lithuania; 20 September 2017 до 22 September 2017; Код 135093

14. Verevkin, N., Lavrentyev, E., Chernyaev, I., Gurin, D., Saint Petersburg State University of Architecture and Civil Engineering, 4 2nd Krasnoarmeyskaya str., Saint Petersburg, Russian Federation. Method of Providing Safe Technical Condition of Vehicles by Technological Design of Enterprises(Conference Paper) Transportation Research ProcediaVolume 20, 2017, Pages 665-670

15. Datsun Y. Determination of the degree of influence of repair processes on serviceability of units of the traction rolling stock(Article). EasternEuropean Journal of Enterprise TechnologiesОткрытый доступVolume 1, Issue 7, 2016, Pages 56-61

16. Shantarenko, S., Kapustyan, M., Supchinsky, O. Omsk State Transport University, Karl Marx Ave., 35, Omsk, Russian Federation Determination of the Electrical Locomotive Energy Efficiency to Evaluate Its Repair Quality (Conference Paper). Volume 692, 2018, Pages 69-7519th International scientific conference Energy Management of Municipal Transportation Facilities and Transport, EMMFT 2017; Khabarovsk; Russian Federation; 10 April 2017 до 13 April 2017; Код 209159

17. Kirihata, T., Uemori, K., Maja, H., Ogura, T., Tokyo Pre-Service Inspection Rolling Stock Depot, Shinkansen Operations Division, Central Japan Railway Company, Japan, Rolling Stock Section, Rolling Stock Department, Shinkansen Operations Division, Central Japan Railway Company, Japan, c. Tokyo Rolling Stock Special Repairs Depot, Shinkansen Operations Division, Central Japan Railway Company, Japan. Study on prevention of failure in passenger door (side sliding door) by using a new statistic method(Article). Japanese Railway Engineering Volume 2018-January, Issue 199, January 2018, Pages 5-8 
18. Zakharov, N.S., Ziganshin, R.A., Nekrasov, V.I., Ziganshina, A.V., Vasilyev, V.I. aFederal State Budgetary Educational Institution of Higher Education, Industrial University of Tyumen (branch in Surgut) Federal State Budgetary Educational Institution of Higher Education, Kurgan State University. Method of planning the needs in spare parts when operating special vehicles(Article). International Journal of Pure and Applied Mathematics Volume 118, Issue 18 Special Issue D, 2018, Pages 3735-3738

19. Bulatov, A.A., Andronchev, I.K., Zheleznov, D.V., Tyapukhin, A.P., Samara State Transport University, Samara, Russian Federation. bGovernment of Samara Oblast, Samara, Russian Federation. Combined approach to an assessment of maintenance of electrical equipment on traction rolling stock(Article). Russian Electrical Engineering Volume 88, Issue 3, 1 March 2017, Pages 140-145

20. Tartakovskyi, E., Ustenko, O., Puzyr, V., Datsun, Y., Ukrainian State University of Railway Transport, Feiierbakh sq. 7, Kharkiv, Ukraine. Systems approach to the organization of locomotive maintenance on Ukraine railways (Book Chapter). Studies in Systems, Decision and ControlVolume 87, 2017, Pages 217-236

21. Ruijters, E., Guck, D., Drolenga, P., Peters, M., Stoelinga, M., University of Twente, EWI-FMT, P.O. Box 217, Enschede, Netherlands. bNedTrain Fleet Services, P.O. Box 2167, Utrecht, Netherlands. Maintenance analysis and optimization via statistical model checking: Evaluating a train pneumatic compressor (Conference Paper). Lecture Notes in Computer Science (including subseries Lecture Notes in Artificial Intelligence and Lecture Notes in Bioinformatics)Volume 9826 LNCS, 2016, Pages 331-34713th International Conference on Quantitative Evaluation of Systems, QEST 2016; Quebec City; Canada; 23 August 2016 до 25 August 2016; Код 179259 